\title{
COLOR APPEARANCE MODEL (NAYATANI MODEL NEWLY REVISED)
}

\section{Yoshinobu Nayatani \\ Professor of Management Engineering Department, Osaka Electro-Communication University}

\section{INTRODUCTION}

In the present article, reported are the special features of the color appearance model developed by Nayatani and his colleagues. 1,2 The model was newly revised on its chroma and hue determinations. 3,4 Their methods and effectiveness are also introduced.

\section{SUMMARY OF THE REVISED MODEL}

The revised model is described by eq.(1) at the opponent-colors stage. The responses $Q, S_{R G}$, and $S_{Y B}$ in eq.(1) were transformed from the three responses $U_{R}$, $U_{G}$, and $U_{B}$ corresponding to the nonlinear cone outputs in the first stage of the model.

$$
\begin{aligned}
& Q=k_{Q}\left[(2 / 3) e(R) U_{R}+(1 / 3) e(G) U_{G}\right], \\
& S_{R G}=k_{C} E_{S}(\theta)\left[U_{R}-(12 / 11) U_{G}+(1 / 11) U_{B}\right], \\
& S_{Y B}=k_{C} E_{S}(\theta)\left[(1 / 9) U_{R}+(1 / 9) U_{G}-(2 / 9) U_{B}\right],
\end{aligned}
$$

where $e(R)$ and $e(G)$ in the $Q$ response are the constants for making the $Q$ response correlate well with the CIE 1976 metric lightness. The coefficients $k_{Q}$ and $\mathrm{kC}_{\mathrm{C}}$ in eq.(1) are scaling constants. From eq.(1), lightness $\mathrm{L}^{*}$ and saturation $S$ are derived by

$$
\begin{aligned}
& L^{*}=Q+50, \\
& S=\left[S_{R G^{2}}+S_{Y B}{ }^{2}\right]^{1 / 2} .
\end{aligned}
$$

Further, chroma $\mathrm{C}$ is given by

$$
\mathrm{C}=\left(\mathrm{L}^{*} / 50\right)^{0.7} \mathrm{~S} \text {. }
$$

The latest revision of the model consists of the following three points.

(1) The new chroma is specified by $\mathrm{eq}_{*}(4)$. The chroma previously used corresponds to the saturation of eq.(3).

(2) As the coefficient for correcting the eccentricity of chroma and saturation scales, $E_{S}(\theta)$ corresponding to the chromatic strengths of spectral colors at hue angle $\theta$ are introduced instead of $\mathrm{e}_{S}(\theta)^{5}$ previously used.

(3) The method of the hue determination is also revised for the change from $e_{S}(\theta)$ to $\mathrm{E}_{\mathrm{S}}(\theta)$. 


\section{SPECIAL FEATURES OF THE MODEL}

Irrespective of the new revision, the model can predict the color appearances of object colors on various achromatic backgrounds under various illuminations, and it has the following special features.

(1) Different types of functions are used to account for the effect of adapting illumination and for the change of test color stimulus. The former is predicted by the function similar to a physiological-response function of retina, and the latter by the logarithmic function normalized by its background. This structure of the model introduces the following characteristics.

(a) The model gives the opponent-colors responses including whitenessblackness response in its fundamental form instead of lightness.

(b) The Helson-Judd effect and the Stevens effect are latently implied in the model.

(c) A similarity holds between the Retinex theory and the Nayatani model in their formulations. The model may be applied to predict the color appearances of object colors in a complex visual field.

(2) The chromatic adaptation transform studied by CIE TC 1.32 is directly derived from the model. Both models are completely compatible to each other. This is practically convenient.

(3) The lightness $L^{*}$ in eq.(2) is very similar to the CIE 1976 metric lightness.

(4) By adding a further analysis, the Nayatani model can predict various color vision phenomena pretty well, such as the Helmholtz-Kohlrausch effect, the colorimetric purity discrimination, and the zero-grayness function $G_{0}(\lambda)$ by Evans. 6

\section{REVISION OF CHROMA SCALE}

\subsection{Adoption of $E_{S}(\theta)$}

Figure 1 shows $E_{S}(\theta)$ adopted. Its wavelength dependency $E_{S}(\lambda)$ in Fig.2 is similar to the function $G_{0}(\lambda)$. As $E_{S}(\theta)$ has the concept of chromatic strength of spectral colors (including colors on the red-purple locus), it is very rational to use the function similar to $\mathrm{G}_{0}(\lambda)$.

By using $E_{S}(\theta)$, constant Munsell-Chroma or NCS-chromaticness loci are almost circular on the $C_{R G}-C_{Y B}$ plane of the model. Figure 3 shows the results of the Munsell scheme.

\subsection{Introduction of Lightness Dependency of Chroma}

In the chroma previously used in the model (the saturation $S$ after the revision), the size of a constant Munsell chroma locus decreases by raising the lightness of test object colors. The new chroma in eq.(4) gives almost the same size to constant Munsell-Chroma loci at Munsell Values $8 \%, 5 /$, and 3/. The loci are shown in Fig.3.

\section{HUE DETERMINATION}

The coefficient $E_{S}(\theta)$ represents the chromatic strength at hue angle $\theta$, and it can be used for determining hue perceptions as same as $\mathrm{e}_{S}(\theta)$. 


\subsection{Equation for Hue Determination}

The following equation holds between perceived hue difference $\Delta H$, its corresponding hue-angle difference $\Delta \theta$, and $E_{S}(\theta)$ at $\theta$.

$$
\Delta \theta=k\left[E_{S}(\theta)\right]^{2} \cdot \Delta H,
$$

where $\mathrm{k}$ is a constant. In case of $\Delta \mathrm{H}=$ constant, $\Delta \theta$ is proportional to the squared chromatic strength $\left[\mathrm{E}_{S}(\theta)\right]^{2}$.

The relation may be considered a differential form of the concept of hue determination using $e_{S}(\theta)$.

\subsection{Results}

The methods using $\mathrm{e}_{S}(\theta)$ and $\mathrm{E}_{S}(\theta)$ are compared in the hue range between unique blue (B) and red (R). The relation between $\theta_{C}$ and $\Delta \theta$ is shown in Fig.4 to each of the eight hue steps equally divided between (B) and (R). $\theta_{C}$ is the hue angle of the center of each hue step $\Delta \mathrm{H}$, and $\Delta \theta$ is the hue-angle difference corresponding to $\Delta \mathrm{H}$.

The line with dots in Fig.4 corresponds to the previous method using $\mathrm{e}_{S}(\theta)$, and the curve with open circles to the method using $E_{S}(\theta)$. In the method using $\mathrm{e}_{S}(\theta), \Delta \theta$ for the constant hue step becomes small by moving from unique blue to red. To the contrary, in case of using $E_{S}(\theta), \Delta \theta$ becomes small near both unique hues, and large at their intermediate hues $\left(\theta=270^{\circ}\right.$ to $\left.300^{\circ}\right)$.

\subsection{Munsell Hue Step}

The relation of $\Delta \theta$ and $\theta_{C}$ of the Munsell scheme is shown in Fig.4 by the dotted line with open triangles to the eight hue steps between $5 B$ and $5 R$ with hue notations 5 and 10 at $5 / 2$. The results show a very good agreement with those using $\mathrm{E}_{S}(\theta)$. The analysis on the NCS hues also supported $\mathrm{E}_{S}(\theta)$.

\section{CONCLUSION}

The special features of the Nayatani model were described from the view points of its theoretical formulations and its applications to various color vision phenomena. In addition, the latest revision of the model was introduced.

The revised Nayatani model correlated very well with the Munsell and the NCS schemes. This is a good field trial to the model. The methods of chroma and hue determinations using $E_{S}(\theta)$ have the same common concept (the chromatic strengths of spectral colors) as the Helmholtz-Kohlrausch effect and the $G_{0}$ function by Evans, and they are generally applicable to any other color appearance models.

1. Y.Nayatani, K.Hashimoto, K.Takahama, and H.Sobagaki, A nonlinear colorappearance model using Estevéz-Hunt-Pointer primaries, Color Res. Appl. 12, 231-242(1987).

2. Y.Nayatani, K.Takahama, H.Sobagaki, and K.Hashimoto, Color-appearance model and chromatic-adaptation transform, Color Res. Appl. 15, 210-221(1990).

3. Y.Nayatāni, Revision of Chroma and Hue Scales of a Nonlinear ColorAppearance Model, to be submitted to Color Res. Appl. (1994).

4. Y.Nayatani, Lightness Dependency of Chroma Scales of a Nonlinear ColorAppearance Model, to be submitted to Color Res. Appl. (1994). 
5. R. W. G. Hunt, A model of colour vision for predicting colour appearance, Color Res. Appl. 7, 95-112(1982).

6. Y.Nayatani, H.Sobagaki, and K.Hashimoto, Relation on Helmholtz-Kohlrausch effect, purity discrimination, and $\mathrm{G}_{0}$. function, Proc. of the 7 th Congress of the International Colour Association, Vol. B, Budapest, Hungary, 13-18 June 1993, pp. CO3·12-CO3·15, Hungarian National Colour Committee, 1993.

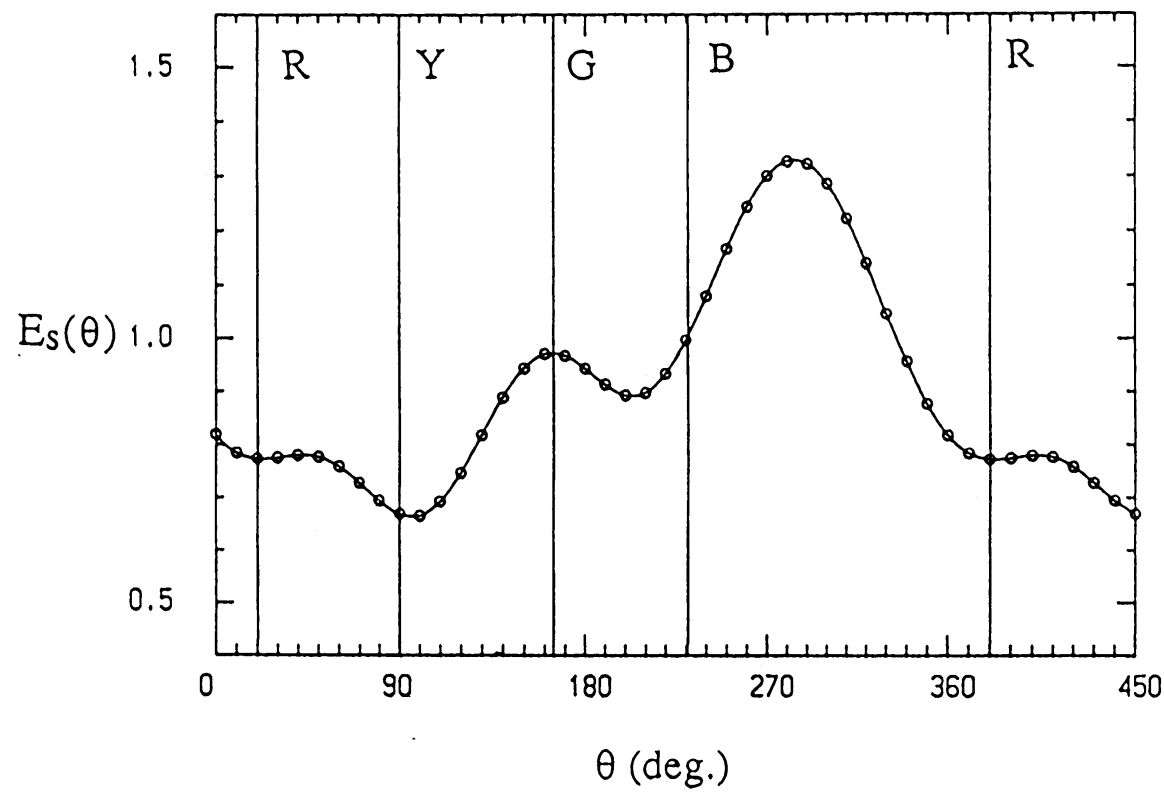

Fig.1 The new chromatic-strength function $E_{S}(\theta)$ as a function of hue angle $\theta$. Vertical lines corresponding to unique hues.

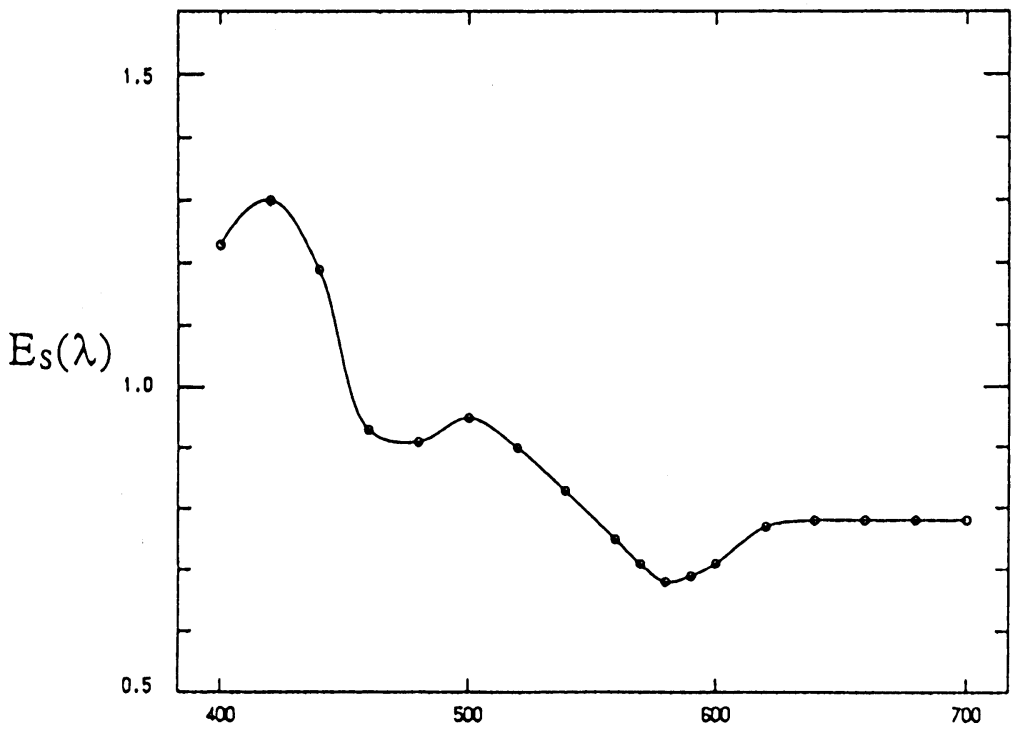

$\lambda(\mathrm{nm})$

Fig.2 Spectral characteristic of the chromatic strength function $E_{S}(\lambda)$ newly proposed. 


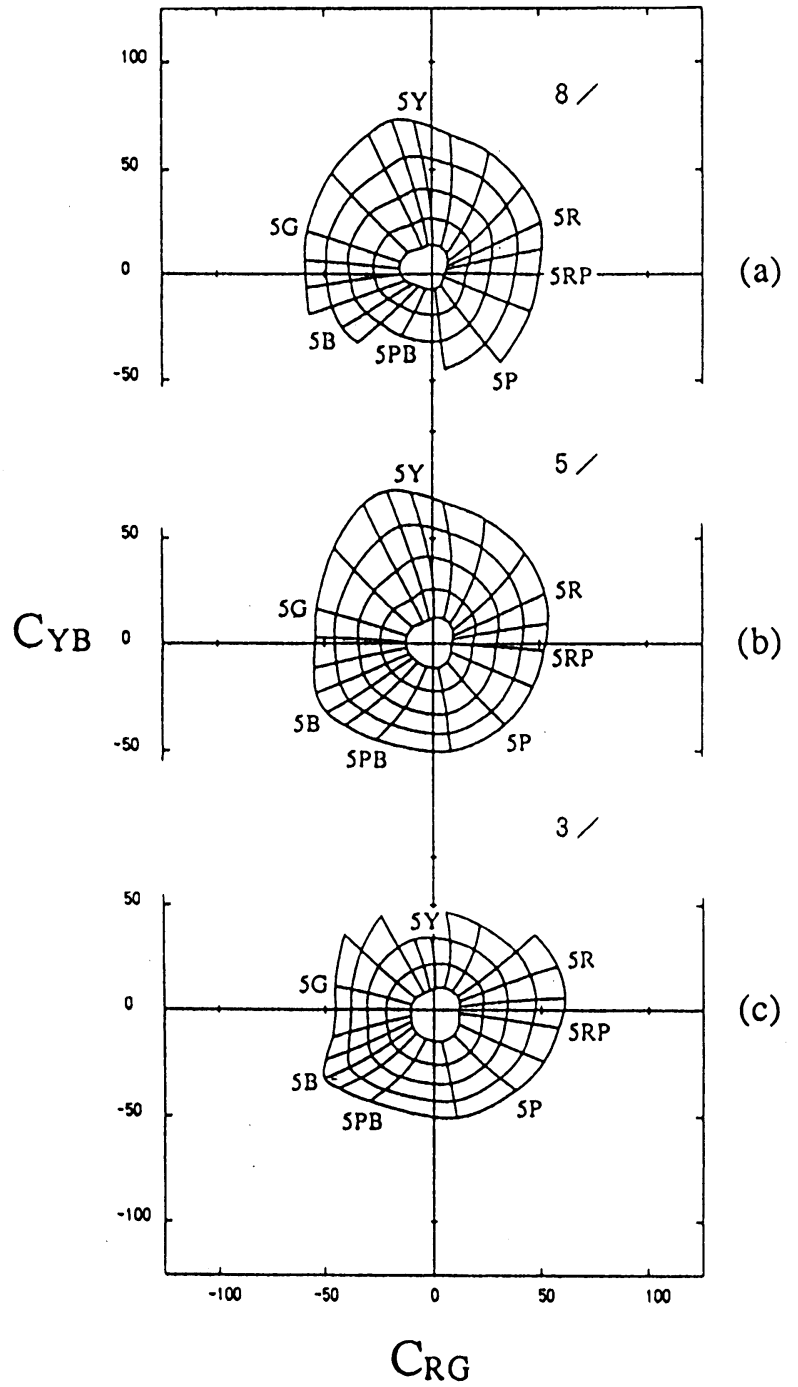

Fig.3 Equal Munsell Hue and Chroma loci at Munsell Values $8 /$ in (a), 5/ in (b), and $3 /$ in (c) on the $C_{R G}-C_{Y B}$ plane of the nonlinear color appearance model.

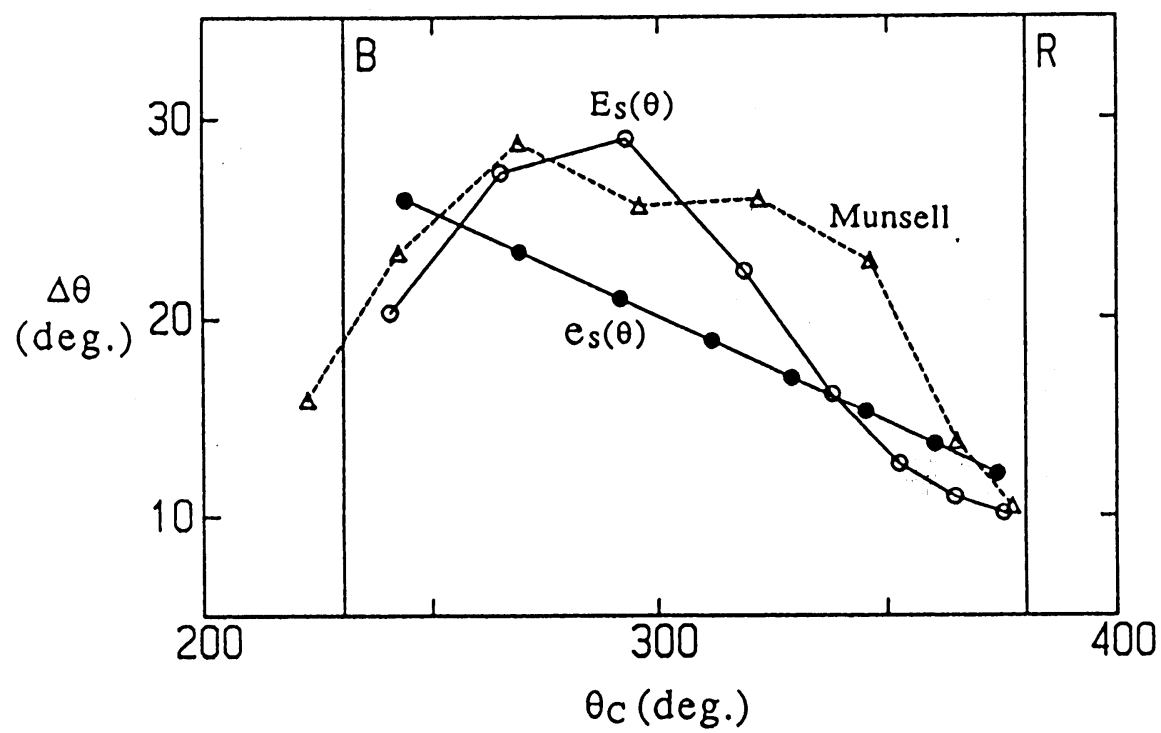

Fig.4 Relations between $\theta_{\mathrm{C}}$ and $\Delta \theta$.

Dots for $e_{S}(\theta)$, open circles to $E_{S}(\theta)$, and open triangles to Munsell hues. 